\title{
Job SHOP Scheduling MeThods REVIEW
}

\author{
Buchmeister, B., PALCIC, I. \& OJSTERSEK, R.
}

Abstract: A production system can be viewed as a network through which jobs flow, wait in buffers, and are processed by workstations. One of the main problems in job shops is how to handle the variety of products. The resources used in production are limited, so scheduling becomes a very important concept for management decision making. This importance draws the attention of both practitioners and academicians to scheduling. Scheduling is a complex and difficult problem. Scheduling the production of multiple products is a classic problem in production planning research. Long lead times, unacceptable work-in-process (WIP) inventories, failure to meet due dates, and other barriers to profitability are found in every manufacturing facility. There are job sequencing methods, resource allocation rules, and mathematical and heuristic algorithms. Conventional optimization methods are insufficient for large problems in terms of solution time. In this paper, an overview of scheduling methods for a job shop and a discussion about their applicability are given.

Key words: Scheduling methods, Job shop, Resource allocation, Problem complexity, Optimization algorithms
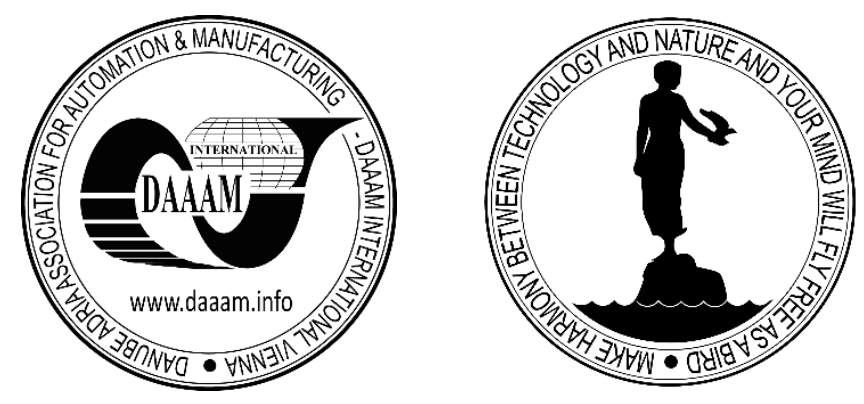

Authors' data: Prof. Dr. Sc. Buchmeister, B[orut]; Prof. Dr. Sc. Palcic, I[ztok]; Dr. Sc. Ojstersek, R[obert], University of Maribor, Faculty of Mechanical Engineering, Laboratory for Production Management, Smetanova 17, 2000 Maribor, Slovenia, borut.buchmeister@um.si, iztok.palcic@um.si, robert.ojstersek@um.si

This Publication has to be referred as: Buchmeister, B[orut]; Palcic, I[ztok] \& Ojstersek, R[obert] (2021). Job Shop Scheduling Methods Review, Chapter 01 in DAAAM International Scientific Book 2021, pp.001-020, B. Katalinic (Ed.), Published by DAAAM International, ISBN 978-3-902734-31-0, ISSN 1726-9687, Vienna, Austria

DOI: $10.2507 /$ daaam.scibook.2021.01 


\section{Introduction}

The job shop scheduling problem is a subfield of production scheduling that is one of the most difficult combinatorial optimization problems (Ojstersek et al., 2020). A typical job shop production has jobs with low production volume and high diversity (HMLV - high-mix, low-volume) and simultaneously processes several different orders (jobs) in small product quantities with common, limited capacities. Work orders have different workflows, delivery times, priorities, quantities, and requirements for materials and necessary resources for processing (Velaga, 2018). Scheduling goals are often conflicting and constrained, and actual production is hindered by a number of potential uncertainties and risks (Zhang \& Zhang, 2020).

Job shop production control includes the following functions:

- establish deadlines and times for production release of incoming customer orders with different workflows depending on the existing workload and availability (occupancy) of resources and materials,

- planning of required materials for each order, and

- scheduling of production.

All three functions are interdependent, interrelated, and cannot be performed independently (Velaga, 2018). Production management problems are exacerbated by a number of factors including: significant changes in customer orders, changes in due dates and priorities, delays in delivery of materials, repairs/rejections due to poor quality, machine breakdowns, acceptance of rush orders for higher profits, etc.

More and more products tailored to the customer create more job shops in the manufacturing world. Make-to-order (MTO) and engineer-to-order (ETO) production systems belong to the job shop class in terms of production complexity. In general, a job shop is a production unit in which unit quantities are usually small, process requirements vary by customer order, job processing begins after receipt of the customer order, and many work orders are processed simultaneously using shared resources. Material and production resource requirements also vary widely in job shop production. Most manufacturing plants with custom products can be classified as job shops. Certain manufacturing plants treat jobs as projects that have the corresponding tasks in the prescribed sequence on limited resources. In terms of production scheduling, these systems are no less complex than HMLV shops. Although most job shops are smaller in terms of capacity and turnover, they are more complex from a production control perspective than a large, repetitive, mass production system. Many job shops with small capacity and low capital have lower raw material inventories, which creates problems in meeting short delivery schedules (Velaga, 2018). Some important features of job shops are:

- The following are not foreseeable: the time of receipt of the customer's order, the due date of the order (if specified by the customer), the order quantity, and the process and material requirements of the order.

- For most customer orders, manufacturing does not begin until after the order is received, so it is unacceptable to have final products ready for future demand. This is the case with the MTO and ETO production systems mentioned earlier. 
- The material procurement process for a customer order can begin after the order is received.

- Using shared finite-capacity resources, a job shop simultaneously processes numerous jobs with different routings, quantities, due dates, priorities, and material and resource requirements.

- Multiple jobs can wait in a queue for a resource at a workstation.

- Product mix and bottlenecks change frequently over time (Velaga, 2018).

This complexity makes it difficult to accurately predict manufacturing order progress, WIP inventory at each work centre, bottleneck formation, resource utilisation, manufacturing throughput, and completion times. The bottlenecks can shift between workstations due to the changing product mix. In job shops, it is not easy to perform proactive capacity planning to avoid bottlenecks and improve workflow, production lead times, on-time delivery, and manufacturing performance.

Each of the above factors can impact workflow, bottlenecks, WIP, lead times, ontime deliveries, critical resource utilisation, and shop throughput. Many make-to-order manufacturers set due dates for customer orders, if customers allow, based on certain average lead times without regard to the current situation. Similarly, they set production release times based on due dates and average lead times. Resourceconstrained production scheduling is necessary to control complex job shop production and predict the flow of work, the formation of bottlenecks, and the completion times of orders. The control of complex production is not effective without predicting the workflow and what-if analysis of production (Velaga, 2018).

Many make-to-order manufacturers somehow manage their production by making quick, real-time decisions based on experience, intuition, common sense, and simple calculations, pushing each order from one workstation to the next as the order progresses. If an order is delayed, they can take emergency action (firefighting) to avoid late delivery. It is not easy to understand the complexity of job shop scheduling as long as production is somehow controlled by real-time scheduling and firefighting. In daily production meetings, there are serious discussions about the situation, and managers regularly try to solve new problems. There is an opportunity to improve the production performance in make-to-order manufacturing without capacity expansion if the workload is efficiently scheduled. Sensible and systematic production scheduling takes into account demand flow, available resource capacity, inventory levels, material planning, existing workload and cash flow (Velaga, 2018). In the paper we want to present different approaches to solving the job shop scheduling problem, their characteristics, limitations and requirements, and their suitability for use.

\section{Approaches to Job Shop Scheduling}

Nowadays, many approaches such as Finite Capacity Scheduling, CONWIP, Lean Manufacturing, Quick Response Manufacturing (QRM), the Theory of Constraints (TOC), etc. are used for production control and management. All these approaches provide a rough or detailed production schedule either in real time or in advance. The following is a brief overview of some production scheduling methods including the manual effort involved (Velaga, 2018). 


\subsection{Manual Scheduling}

Often, the role of a scheduler is limited to tracking job progress on the shop-floor and reporting to management. There are several job shops where production scheduling is simplified by the following practice (Velaga, 2018):

- Due dates for customer orders are set based on some average lead time (e.g., four weeks), regardless of upcoming workload and resource availability.

- Job loading time is based on the availability of material and a predetermined production lead time. Some people do some simple calculations for workload at critical workstations and adjust job loading times accordingly.

- Each job is pushed from one workstation to the next at the completion of an operation, and a particular dispatch rule is applied at each workstation to select jobs (for processing) from the appropriate queue.

- If a job is in danger of exceeding its due date, it will be processed with high priority, working overtime if necessary.

- The job is delivered after all of its operations have been completed.

Job shop production is manageable with this practise, but it can lead to high WIP, long lead times, poor on-time delivery, and frequent firefighting (Velaga, 2018). In their effort to meet the due dates, manufacturing professionals engage in extensive realtime scheduling, i.e., they engage in "firefighting" without knowing the impact of their real-time decisions on the production schedule. More often than not, the firefight propagates and stays on the shop floor for an extended period of time. Firefights can be a sign of chaos, poor planning, and lack of understanding of the dynamic nature of make-to-order manufacturing. They can weaken management and customer confidence in the shop floor's ability to meet due dates. Job shop cannot readily improve its performance unless the need for firefighting is minimised without sacrificing productivity. Manual scheduling is not efficient for make-to-order production that simultaneously handle numerous, diverse jobs with different due dates. Scientific scheduling can significantly improve the throughput, delivery reliability, and resource utilisation of HMLV job shops.

\subsection{Scheduling on Whiteboards and Excel Spreadsheets}

Many planners still use whiteboards for production scheduling purpose. This simple, manual method might be sufficient for some small production systems. However, it is very inconvenient and ineffective for scheduling numerous operations with different orders on resources with limited capacity. It is also not helpful for quickly rescheduling the workload when the actual workflow deviates significantly from the whiteboard schedule (rush orders, rework, machine breakdown, etc.). This is a static decision support tool trying to manage a dynamic problem!

Many software vendors have extended the whiteboard concept by offering an electronic version that facilitates manual creation of schedules and changes to the Gantt chart (on the computer screen). Inexpensive software tools that facilitate production planning at the shop floor level by simply dragging and dropping onto the computer screen are far more useful than whiteboards for dynamic scheduling of job shops. Creating production schedules in Excel allows you to quickly manipulate data and 
perform basic operations, and most ERP programmes also allow you to export basic work order information as a starting point.

Excel spreadsheets are in demand for production scheduling in job shops for many reasons (Velaga, 2018):

- Almost all people who are familiar with personal computers can easily handle data in Excel spreadsheets.

- Data needed for scheduling can be easily pulled from most ERP / MRP packages into Excel spreadsheets.

- Many data operations like editing, reformatting and transformation can be easily performed in Excel spreadsheets.

- Programming in VBA is useful for advanced operations with Excel data.

- Some people are under the impression that scheduling applications in Excel are cheaper and good enough for small job shops.

They are indeed good enough for small and simple make-to-order manufacturing systems. Despite many such advantages, Excel applications have some major limitations for production scheduling in complex job shops (Oh et al., 2014). Excel cannot simply support a versatile scheduling paradigm and powerful logic to sufficiently handle the scheduling complexity of many HMLV job shop systems, regardless of the size of the organisation. For example, Excel applications are not efficient at handling:

- individual weekly calendars and calendar exceptions of resources,

- changes in order priorities,

- multiple resource requests of operations,

- rapid and comprehensive what-if analyses, etc.

They also lack powerful and user-friendly graphing capabilities and drag-anddrop features, and they offer little help in controlling manual data entry errors. Although users can easily edit Excel files, editing is prone to human error. As the size and complexity of production increases, Excel scheduling applications tend to become very large, cumbersome, and inflexible. These applications are very slow to schedule hundreds of orders in high-mix, low volume job shops (Velaga, 2018).

However, most of these disadvantages can be avoided if the scheduling software can take data directly from Excel spreadsheets. A small add-in software component may be required if the Excel data is not in the desired format. For small job shops, there is no guarantee that Excel-only applications with sufficient scheduling capabilities will have lower operating costs (including training and implementation) than some established scheduling tools.

\subsection{Scheduling by Project Management Software Tools}

Job shop management is similar in many respects to resource-constrained multiproject management. Among the most important differences are the following:

- In many job shops, the dependency relationships between tasks in a job can be expressed by a task sequence, whereas describing the dependency relationships between tasks in a project usually requires a directed network.

- In most job shops, a task cannot be started until all previous tasks have been completed. In a project environment, it is not essential that all previous tasks be 
completed before a task is started. However, the task cannot start until all previous tasks have started.

- Tasks in job shops are less likely to be effort-driven, which means that it may not be possible to shorten the duration of tasks by allocating more resources.

- Tasks in projects have longer durations with multiple changes.

- Projects are more likely to be affected by major uncertain events (Velaga, 2018).

The best known methods for project scheduling are CPM (Critical Path Method) and PERT (Program Evaluation and Review Technique). CPM is used for projects with deterministic (fixed) activity durations, while PERT is used for projects with probabilistic activities (optimistic, most likely, pessimistic time).

Many job shop schedulers use project management (PM) tools for production scheduling. When jobs queue and compete for resources with finite capacity, the critical path method (a project scheduling method adopted by PM software) can create meaningless production schedules that planners helplessly accept (Velaga, 2018). The vast majority of PM packages are unsatisfactory for resource-constrained scheduling of thousands of tasks because they are unable to resolve resource conflicts automatically. It is usually tedious to schedule and reschedule numerous orders (with hundreds of tasks) in a workable manner when all resource conflicts in the schedule must be resolved manually by drag-and-drop operations, as required by project management packages. These tools do not provide a powerful, reliable, and fast whatif analysis or a capacity planning mechanism for production systems. However, the common project management tools are excellent information systems with an elegant graphical user interface that provides a very effective representation of project/production schedules (Kong \& Dou, 2021; Chakrabortty et al., 2021). Most PM tools are not powerful enough for resource-constrained, complex production scheduling with thousands of tasks. Most users of such tools in the production environment do not know that powerful tools exist for resource-constrained scheduling.

\subsection{Scheduling Modules of ERP Systems}

In the manufacturing sector today, there has been rapid progress in the adoption of sophisticated enterprise-wide integrated information systems known as Enterprise Resource Planning (ERP) systems. Companies are able to manage all their information very easily and improve the efficiency of their business operations with the help of these systems. The usefulness of ERP systems has further increased in manufacturing by integrating shop floor data collection systems with a central database. From a customer relations perspective, these systems allow companies to communicate realtime order status to their customers (Velaga, 2018). However, many ERP systems do not quite live up to the term "resource planning" as they lack strong planning and intelligent decision support modules.

There are several ERP and shop management software packages that promise to eliminate all the disadvantages of the scheduling methods described above. The ERP scheduling modules are usually better than whiteboards and Excel spreadsheets, but are still inadequate or inefficient for complex job shops. Many ERP scheduling modules are currently used by job shops mainly because: 
- they replace the tedious manual scheduling and rescheduling done on whiteboards and in Excel spreadsheets,

- there is no need to to provide order information for planning and scheduling separately, and

- the level of knowledge about scheduling in operations is low (Velaga, 2018).

The modules based on MRP scheduling logic are unsuitable for job shop scheduling. Many of them are often unable to automatically generate a feasible schedule that satisfies all relevant constraints. Some of them determine the waiting times at the different workstations in advance (estimation) and use these times when creating the schedule. It is almost impossible to implement such modules on the shop floor. They perform infinite capacity scheduling and determine the required capacity needs, which is not always practical. In these modules, an electronic whiteboard may be available to manually fix an infeasible schedule through drag-and-drop operations. Some ERP planning modules create an infeasible schedule with resource conflicts and export it to a PM tool, requiring a scheduler to manually repair the schedule through drag-and-drop operations on the Gantt chart. This approach is very tedious when it comes to rescheduling a large number of jobs and performing what-if analyses in response to significant changes in production. The modules that require a lot of execution time to create a single feasible schedule are not efficient for quick and comprehensive what-if analysis. Interestingly, some job shops download production data from ERP systems and schedule production in an Excel spreadsheet or Access application (Velaga, 2018).

\subsection{Scheduling by Theory of Constraints (TOC)}

One of the simple methods proposed for production scheduling in complex job shop is the Theory of Constraints (TOC) method Drum-Buffer-Rope (DBR). It was developed by Eliyahu Moshe Goldratt, an Israeli management guru, in the early 1980s when companies did not have sophisticated information systems, plant data collection mechanisms, powerful and inexpensive computers, and affordable scheduling tools. After development activities on software tools for production scheduling, Goldratt and Cox (1986) recognised the difficulty of scheduling complex production systems under these unfavourable conditions and the need to simplify these complex systems so that the scheduling difficulties could be eliminated.

The DBR scheduling method is based on the fundamental assumption that a production system has a single resource constraint and the other resources have sufficient capacity to fully support any feasible schedule for the constrained resource. When this assumption is not true, management must select one resource as the constraint and increase the capacity of the other resources to ensure the applicability of the simple DBR scheduling method. The constrained resource may change over time. DBR is quite simple to understand and practice, and a scheduler can easily implement it with an Excel spreadsheet. From an execution perspective, DBR involves a procedure called buffer management that accounts for system variations (Velaga, 2018).

The underlying assumption of DBR that there is only one committed resource (bottleneck) allows us to schedule jobs on the committed resource (in ascending order of due dates), ignoring the capacity of all uncommitted resources. Job loading times 
are determined in accordance with the schedule developed for the bottleneck resource, roughly taking into account the effects of uncertainty. The basic idea is to keep the constraint resource always busy while controlling the WIP in the system by releasing jobs at the right time. DBR can be practically effective only if the underlying assumption is true. If the assumption is not true, the job queues caused by finite capacity resources will adversely affect the buffer management in DBR. Modifying a complex job shop to support the implementation of the simple DBR scheduling method can sometimes be quite expensive and requires strategic thinking, as such changes can result in excessive resource capacity. It is reasonable to keep production scheduling simple in complex systems, but it is important to consider the costs we must incur to make this possible (Velaga, 2018; Buchmeister et al., 2019).

\subsection{Scheduling by Lean Manufacturing Practices}

The Toyota Production System (TPS) evolved over decades into an inspiring world-class production system thanks to the continuous, dedicated efforts of Toyota employees. Toyota developed many methods, concepts, practices, principles and work culture that are very effective in manufacturing. However, some of the concepts and methods such as Heijunka, takt time, one-piece flow, and Kanban-based production control are not very useful in job shops with simultaneous production of various lowvolume jobs (Velaga, 2018). The TPS has spread globally through its philosophy of production improvement and waste reduction. However, despite the efforts of organizations to adapt to the new system, it is not yet possible to fully implement it in organizations (Callefi \& Crubellate, 2020; Gavriluta, 2019).

The Kanban system developed by Toyota is very powerful for controlling repetitive production. It can be considered as a real time production scheduling system. In repetitive production, it prevents starvation of bottleneck workstations and creation of large inventories at each workstation by regulating the flow of materials through the production system (Lendinez, 2019). The Kanban system captures uncertainty, natural variation, and differences in production rates at workstations, but it is most effective when demand is consistent and predictable. However, in job shops with unpredictable demand and different orders passing through different sequences of workstations, no Kanban system can provide predictability of workflows, WIP, lead times, resource utilisation patterns, and order completion times. Such predictability is essential for capacity planning and production control in complex job shops with heterogeneous workloads. The Kanban system cannot provide what-if analysis of dynamic production as required by job shops. Also, with a Kanban system, it is not easy to determine the right time to load a job into the shop based on the due date, quantity and routing of the job, resource availability, and the current workload in the shop. Kanban control does not provide a comprehensive understanding of the dynamic nature of job shops. Similarly, Heijunka scheduling from TPS is not relevant for job shops that simultaneously produce many low-volume jobs with varying due dates, priorities, routings, process times, and resource and material requirements (Velaga, 2018).

Since Toyota demonstrated the power of Heijunka scheduling and Kanban control in managing its volume production, there have been many attempts to forcibly implement it with false confidence for production scheduling and control in complex 
job shops. In job shops with simultaneous production of diverse orders, a Kanban system will be quite cumbersome and have little impact on throughput and on-time delivery. Quick Response Manufacturing (QRM) is another approach to manufacturing control that can also be considered as real-time job shop scheduling. The POLCA method of QRM is better suited than Kanban control to handle the complexity associated with multiple job routings. However, POLCA also cannot provide the necessary what-if analysis and predictability required by job shops (Velaga, 2018).

\subsection{Finite Capacity Scheduling (FCS)}

Finite Capacity Scheduling (FCS) tools are also available for job shop scheduling. However, the academic literature shows a gap in research and practise in this area due to the lack of studies that investigate the implementation of production scheduling solutions in real-world contexts (Carvalho et al., 2014). The main drawbacks of FCS are:

- users have to define numerous parameters of the tool,

- users perceive the tools as quite cumbersome, unfriendly and not very intuitive,

- the users need a lot of training for the tools,

- the input data is either unavailable or incorrect, and

- the tools need a lot of computing power and time (Velaga, 2018).

However, with the rapid advancement of hardware and software, these tools have improved tremendously over time and have overcome their drawbacks. The Windowsbased tools offer the user a lot of flexibility, convenience and context help. The highspeed personal computers make it much easier to use the FCS tools. As top management begins to embrace the schedule output of these tools, data quality and availability will begin to improve.

With the availability of powerful information systems and sophisticated operational data collection systems, job shops are able to track the status of jobs and make seemingly rational decisions in real time to meet job due dates. FCS tools depend on these same systems to create efficient production schedules and display the dynamic workflow and the formation of bottlenecks over time. Implementing FCS tools brings many benefits to job shops, such as quick rescheduling of workload, prediction of future bottlenecks and job completion times, and efficient capacity planning. FCS also has some disadvantages. Each FCS tool works with a particular scheduling model. If a scheduling problem does not fit into the underlying paradigm, FCS 's solution might be meaningless, even if it seems very sophisticated. It may be impractical to implement FCS solutions derived purely on the basis of available hours for each resource in each time interval (day, week, month, etc.) on the shop floor. Such schedules may not specify the sequence of jobs to be loaded at each workstation. Two major criticisms of FCS are its lack of ability to handle variation and its detailed schedule output. FCS logic can be suitably modified to create time buffers (as in TOC methods) for jobs in some phases to absorb the effect of changes on the schedule. The schedule output can be viewed at the desired level of detail (Velaga, 2018). The schedulers need to use some common sense and domain knowledge while implementing these powerful tools. They can use the tools interactively to get the best schedules. 


\subsection{Mathematical Programming}

Mathematical programming has been applied extensively to job shop scheduling problems (Blazewicz et al., 1991). The problems have been formulated using integer programming, mixed-integer programming, and dynamic programming. The use of these approaches is limited because scheduling problems belong to the class of NP complete problems. The analysis of mathematical programming models for some hard combinatorial problems has provided important computational advances for scheduling problems.

\subsection{Expert/Knowledge-Based Systems}

Expert and knowledge-based systems consist of two parts: a knowledge base and an inference engine that operates on that knowledge base. Formalizations of the "knowledge" that human experts use - in the form of rules, procedures, heuristics, and other kinds of abstractions - are held in the knowledge base, where three types are included: procedural, declarative, and meta knowledge. The first is domain-specific problem-solving knowledge. Declarative knowledge provides the input data that defines the problem domain. Meta-knowledge is the knowledge of how the procedural and declarative knowledge can be used to actually solve the problem. Various data structures have been used to represent the knowledge in the knowledge base, including semantic nets, frames, scripts, predicate calculus, and production rules. The inference engine selects a strategy to apply to the knowledge base to solve the problem at hand. It can be forward chaining (data-driven) or backward chaining (goal driven).

High flexibility and responsiveness to capacity adjustments are key to successful production planning and control in job shops. Knowledge-based systems are very important to achieve production goals (Denkena et al., 2019). Applications of knowledge-based expert systems to scheduling problems also exist in service systems (Lopez-Santana \& Mendez-Giraldo, 2016).

\subsection{Scheduling Algorithms}

Researchers have contributed much literature on job shop scheduling that can be found in research journals and in books. They have developed good concepts and considered interesting goals and constraints of job shop scheduling (Dave \& Choudhary, 2016; Yu \& Lee, 2018). The objectives deal with issues such as WIP, production lead time, job tardiness, unpunctuality, etc. However, the scheduling models and algorithms they developed are largely inappropriate for the actual scheduling problems of job shops. This is mostly because (Velaga, 2018):

- the available mathematical techniques are are inadequate to deal with practical scheduling problems,

- many assumptions are made to reduce a problem to a level where it can be solved by a non-trivial method,

- developing simple methods (heuristics) for reasonably good solutions to complex practical scheduling problems is not an exciting activity for researchers,

- researchers have a natural tendency to take a complex, rigorous view of problems.

The powerful concepts developed by researchers are buried in the mathematical treatment. An overview of the procedures is shown in Fig. 1 (Parveen and Ullah, 2010). 


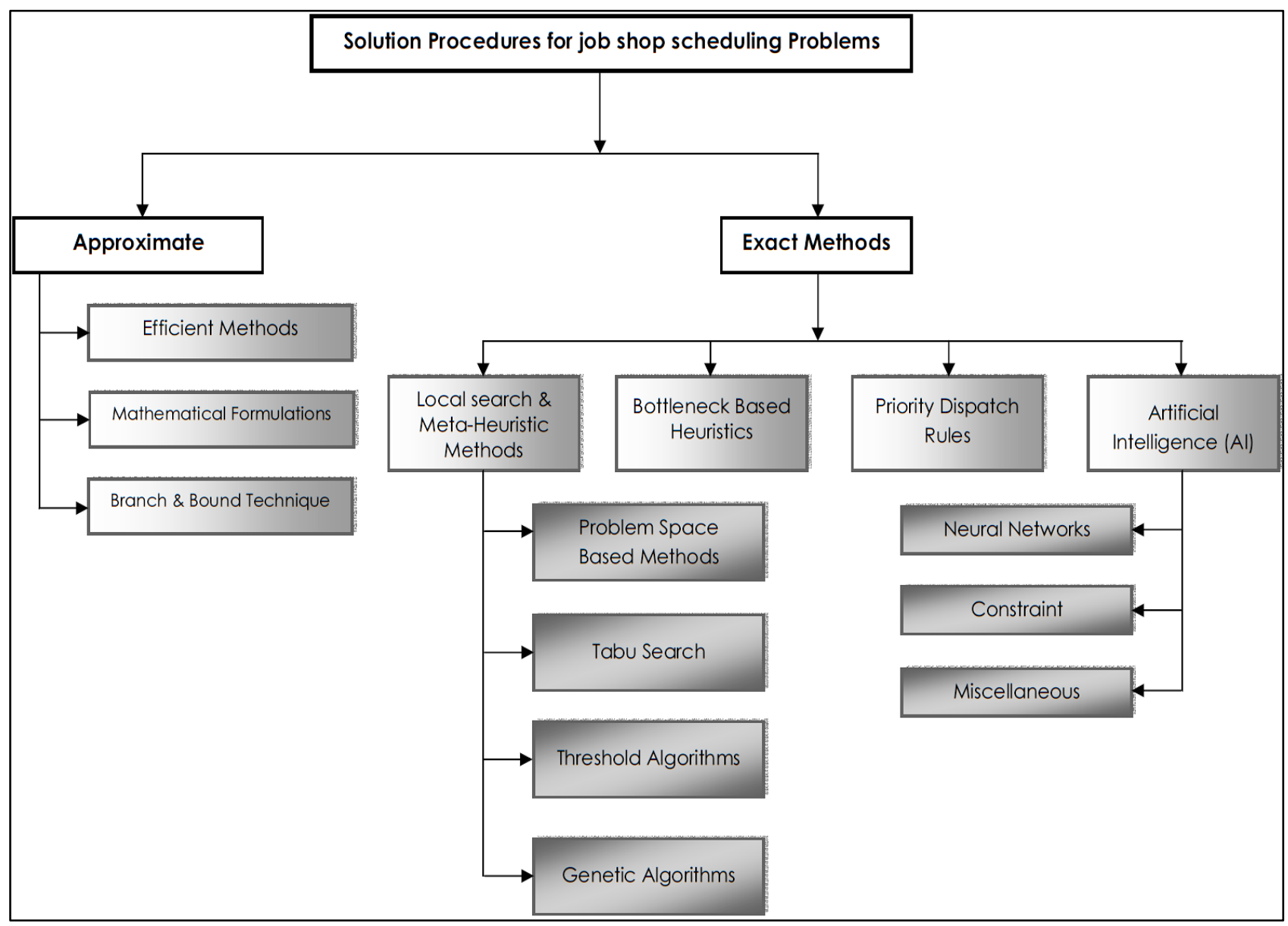

Fig. 1. An overview of solution procedures for job shop scheduling problems (Parveen \& Ullah, 2010).

Solution procedures are usually based on heuristic approaches. These methods provide solutions in a reasonable amount of time, but they do not guarantee the optimal result. They find the near optimal solutions that are satisfactory for large and complex problems (Yagmahan and Yenisey, 2009). Recent studies mainly focus on metaheuristic algorithms. These metaheuristic algorithms are influenced by events in nature and are inspired by the behaviour of animals such as ants or swarms, biological entities such as neurons or genes, or a physical event such as annealing. They use principles from nature to find solutions. Using this natural understanding is one of the main goals of artificial intelligence to make computers more useful in problem solving.

In the following, we will focus on some selected algorithms implemented for scheduling problems, such as simulated annealing, tabu search, genetic algorithms, ant colony optimization, particle swarm optimization, differential evolution, and artificial immune systems. Of course, we must also mention some other established algorithms, e.g.: genetic programming, memetic algorithms, variable neighbourhood search, guided local search, gravitational search algorithm, scatter search, harmony search, greedy randomised adaptive search methods, multi-start methods, bee algorithms, firefly algorithm, cuckoo search, cross entropy method, heuristic Kalman algorithm, bacterial foraging optimization, bat algorithm, shuffled frog leaping algorithm, grasshopper optimization algorithm, dragonfly algorithm, etc. 


\section{Simulated Annealing (SA)}

The basic idea comes from the field of metallurgy, in which a solid is first melted and then slowly cooled. The SA algorithm allows for moves that lead to a better solution than the current solution (upward moves) to escape local minima. The probability of making such a move decreases during the search (Ogbu and Smith, 1991; Low et al., 2004). The SA algorithm is summarized in the following text box:

Step 1:

Generate an initial solution $x$

(Yagmahan and Yenisey, 2009)

Select an initial temperature $T_{0}$

$T=T_{0}$

Step 2:

Generate candidate solution $x^{\prime}$ from current solution $x$

if $f\left(x^{\prime}\right)<f(x)$, then set $x=x^{\prime}$

if $f\left(x^{\prime}\right) \geq f(x)$, then set $x=x^{\prime}$ with a certain probability

Step 3:

Update $T$ according to cooling schedule

If stopping condition is met, then stop; otherwise, go to Step 2.

\section{Tabu Search (TS)}

The Tabu Search algorithm was first proposed by Glover (1989). The TS algorithm depends on the following parameters: initial solution, moves, neighbourhood, search strategy, tabu list, aspiration criterion and stopping criteria. The basic idea of this method is to start from an initial solution and then successively move between neighbourhood solutions. In each iteration, a move is made to the best solution in the neighbourhood of the current solution, which may not be an improving solution. Tabus are used to prevent cycles when moving away from local optima by making nonimproving moves. Tabus are stored in the tabu list. In each iteration of TS, a move is assigned to the tabu list when the move is selected to guide the search from the current solution to its neighbouring solution. This move is then not selected for a number of immediately following iterations. The size of the tabu list is limited by the tabu list size. The size of the tabu list can be fixed or variable (Gupta et al., 1999). The structure of the TS algorithm is shown below.

Step 1:

Generate an initial solution $x ; x^{*}=x$

(Yagmahan and Yenisey, 2009)

Initialize the tabu list

Step 2:

While set of candidate solutions $X^{\prime}$ is not complete

Generate candidate solution $x^{\prime}$ from current solution $x$

Add $x^{\prime}$ to $X^{\prime}$ if $x^{\prime}$ is not on the tabu list T or satisfy the aspiration criterion

Step 3:

Find the best candidate solution $x^{*}$ in $X^{\prime}$

if $f(x)<f\left(x^{*}\right)$, then set $x^{*}=x$

Update the tabu list and aspiration conditions

Step 4:

If stopping condition is met, then stop; otherwise, go to Step 2. 


\section{Genetic Algorithm (GA)}

The Genetic Algorithm is a population-based method based on the mechanisms of natural selection and natural genetics (Gen and Cheng, 2000). The GA maintains a population of individuals $P(t)$ for generation $t$. Each individual represents a solution to the problem. These solutions are encoded in chromosomes. Each individual in the population is scored and assigned a fitness value. Then the population undergoes genetic operations to form new individuals. During a number of iterations, this population evolves until a certain stopping criterion is met:

Step 1:

$t=0$

(Yagmahan and Yenisey, 2009)

Step 2:

Form the initial population $P(t)$

$$
\begin{aligned}
& P^{\prime \prime}(t) \leftarrow \text { crossover }(P(t)) \\
& P^{\prime \prime \prime}(t) \leftarrow \text { mutation }\left(P^{\prime \prime}(t)\right) \\
& \text { Evaluate }\left(P^{\prime \prime \prime}(t)\right) \\
& P(t+1) \leftarrow \operatorname{Select}\left(P^{\prime \prime \prime}(t) \cup P(t)\right) \\
& t=t+1
\end{aligned}
$$

Step 3:

If the stopping criteria are met (number of generation), then stop; otherwise, go to Step 2.

The application of genetic algorithms to the scheduling problems has been widely studied (Wang and Zhang, 2006; Nagano et al., 2008; etc.).

\section{Ant Colony Optimization (ACO)}

Ant Colony Optimization has been proposed in the literature as a new metaheuristic approach to solve difficult combinatorial optimization problems. The main idea of ACO metaheuristic is based on the behaviour of real ants that use the pheromone trail for communication and cooperation. The first example of an ACO algorithm is the Ant System (AS) algorithm, proposed by Dorigo et al. (1991) for the Travelling Salesman Problem. Studies then attempted to improve its performance and, as a result, several ACO algorithms have been proposed (Rajendran and Ziegler, 2004; Ying and Liao, 2004). These extensions include Ant Colony System (ACS), Ant-Q, the Max-Min Ant System (MMAS) and Rank Based Ant System. The structure of ACO:

Step 1:

(Yagmahan and Yenisey, 2009) Step 2:

Pheromone trails, heuristic information, and parameters are initialized

Construct a complete solution for each ant

Apply local search process

Update the pheromone trail

Step 3:

If the stopping condition is realized, then stop; otherwise go to Step 2.

Particle Swarm Optimization (PSO)

The Particle Swarm Optimization algorithm is one of the latest population-based optimization methods. It is based on sociological behaviour associated with flocks of 
birds or schools of fish. PSO consists of a swarm of multiple particles, where each particle represents a solution to an optimization problem. Each particle moves at a position in the multidimensional search space with a certain velocity. It moves according to a function of its current position, velocity, and the best objective function value in the search space along iterations. Each particle adjusts its velocity to update the position of each particle. The velocity is added to the position coordinates of the particle. The general PSO algorithm is summarised below (Tasgetiren et al., 2007; Jarboui et al., 2008).

Step 1:

Randomly initialize particle positions and velocities

(Yagmahan and Yenisey, 2009)

For each particle $i$ :

Evaluate the objective function $f(x)$ at current position $X_{i}$

Step 2:

For each particle $i$ :

If $f\left(X_{i}\right) f($ lbest $)<$ then lbest $=X_{i}$ and $P_{i}=X_{i}$

If $f\left(X_{i}\right) f$ (gbest) $<$ then gbest $=X_{i}$ and $G_{i}=X_{i}$

Step 3:

For each particle $i$ :

Update velocity vector $V_{i}$

Update position vector $X_{i}$

Step 4:

If the stopping condition is realized, then stop; otherwise go to Step 2.

\section{Scatter Search Algorithm (SS)}

Scatter Search is a population-based optimization method that has been successfully applied to optimization problems. SS generates a trial set using the seed solutions that correspond to the feasible solutions for the problem under consideration. An improvement method is used to try to improve the trial solutions and update the reference set. A reference set contains the best solutions found so far with respect to the objective function. A subset of solutions is created by combining solutions in the reference set. These newly created subset solutions are improved and used to update the reference set. This search is terminated when the stopping criteria are satisfied. The SS algorithm is summarised below (Blum and Roli, 2003; Saravanan et al., 2008; etc.).

Step 1:

Generate trial solutions from the seed solutions

(Yagmahan and Yenisey, 2009)

Apply the improvement method to produce one or more enhanced trial solutions

Update the reference set

Step 2:

Generate new subsets from the reference set

Combine these subsets to obtain one or more new trial solutions

Apply the improvement method to the trial solutions

Step3:

Step 4:

Update the reference set

If the stopping condition is realized, then stop; otherwise go to Step 2. 


\section{Differential Evolution Algorithm (DE)}

$\mathrm{DE}$ can be classified as an evolutionary optimization algorithm. In a DE algorithm, candidate solutions are represented by chromosomes based on floating point numbers. DE works as follows: first, all individuals are randomly initialized and evaluated. At each generation, the mutation and crossover operators are applied to the individuals to generate a new population. In the mutation process, the weighted difference between two randomly selected population members is added to a third member to generate a mutated solution. Then a crossover operator follows to combine the mutated solution with the target solution to generate a trial solution. A selection operator is applied to compare the fitness function value of the two competing solutions, namely the target solution and the trial solution, to determine who can survive in the next generation. As long as the termination condition is not satisfied, this process is executed (Qian et al., 2009). The basic algorithm of DE is shown below.

Step 1:

(Yagmahan and Yenisey, 2009)

Step 2:

Initialize population

Step 3:

Evaluate the objective values of all individuals, find the best individual

Mutation

Crossover

Selection

Update the best individual

Step 4:

If a stopping criterion is satisfied, then stop; otherwise go to Step 3.

\section{Artificial Immune System (AIS)}

The Artificial Immune System algorithm is an adaptive system, inspired by theoretical immunology and observed immune functions, principles, and models used to solve problems (Yagmahan and Yenisey, 2009).

The main function of the immune system is to recognize disease-causing organisms, called pathogens, defend against invasion, and eliminate malfunctioning cells. Pathogens are not recognized directly by the components of the immune system. Antigens are small parts of the molecules of pathogens that are recognized by the immune system. There are two types of antigens: self and non-self antigens. Non-self antigens are disease-causing elements, while self-antigens are harmless to the body. Two main groups of immune cells are B cells and T cells. B cells can recognize antigens freely in solution, while $\mathrm{T}$ cells require antigens presented by other supporting cells. Both B cells and T cells contain the surface receptors that can recognize antigens. Antigens are covered with molecules that are recognized by the receptor molecules. One antibody is the B cell receptor molecule. When an antigen is recognized by immune cell receptors, the immune system produces antibodies. The binding of an antibody to antigens is a signal to remove disease-causing organisms. There are several selection mechanisms used in AIS algorithms. Negative selection, clonal selection and immune network models are examples. The following text box illustrates the principle of negative selection (de Castro, 2002). 
Step 1:

(Yagmahan and Yenisey, 2009)

Step 2:

Initialize population Step 3:

Evaluate the objective values of all individuals, find the best individual

Mutation

Crossover

Selection

Step 4:

Update the best individual

If a stopping criterion is satisfied, then stop; otherwise go to Step 3.

\subsection{Neural networks}

Neural networks, also called connectionist or distributed parallel processing models, have been studied for many years in an attempt to mimic the learning and predictive abilities of humans. Neural network models are distinguished by network topology, node properties, and training or learning rules.

Neural networks are organised in a framework based on the brain structure of simple living entities. An artificial neural network provides us with the ability to simulate the network of neurons that make up a human brain, enabling it to learn things from experience and make decisions and predictions in a human-like manner. In these techniques, information processing is performed by a massively interconnected network of parallel processing units. Their simplicity, along with their ability to perform distributed computation and their propensity to learn and generalise, has made neural networks a popular method, allowing them to be used in many real-world applications (Chen et al., 2020), in environments far more complicated than those in a traditional job shop.

Cheung (1994) describes some of the main neural network architectures used to solve scheduling problems: searching network (Hopfield net), error-correcting network (Multi-Layer Perceptron), probabilistic network (Boltzmann machine), competing network, and self-organising network.

Rabelo (1990) was the first to use back-propagation neural networks to solve job shop scheduling problems involving multiple order types with different arrival patterns, process plans and lot sizes. Training examples were generated to train the neural network to select the correct characterization of manufacturing environments suitable for different scheduling strategies and the selected performance criteria. To generate training examples, a performance simulation of the scheduling rules available for the manufacturing system was performed. The neural networks were trained for problems with 3, 4, 5, 8, 10 and 20 machines. To perform this training, a special input feature space was developed. This space contained both job characteristics (such as types, number of jobs in each type, routings, due dates, and processing times) and shop characteristics (such as machine capacities). The neural network output represented the relative ranking of available dispatching rules for this specific scheduling problem and the selected performance criteria. The neural networks were tested in numerous problems and their performance (minimising mean tardiness) was always better than any single dispatching rule ( $25 \%$ to $50 \%)$. 


\subsection{Hybrid systems}

Over the years, researchers have attempted to combine the best features from various approaches described previously to derive more powerful hybrid implementations (which are superior to the individual approaches). The list of hybrid systems includes:

- genetic algorithm and local search,

- genetic algorithm and tabu search,

- neural networks and tabu search,

- etc. (Onwubolu, 2002; Zhang \& Wu, 2010).

Researchers also hybridize approaches such as genetic algorithms, neural networks, tabu search, and ant systems with other good heuristics to generate better initial solutions (instead of random generation). This drastically improves the global solutions obtained.

\section{Conclusion}

Production scheduling is an important part of production management in complex job shops. Many do not seem to pay serious attention to this decision-making function, which plays a central role in linking demand, resource availability, material planning, finances, and inventories (T'kindt \& Billaut, 2002). Its role is vital in job shops with high-mix, low-volume production. Researchers consider scheduling as one of the most challenging of all decision problems, but the available knowledge about scheduling in manufacturing industry is low (Velaga, 2018). Practical aspects of scheduling are not formally taught in universities and training on scheduling in industry is almost nonexistent. People learn production scheduling through common sense, experience, and software tools. The understanding of the dynamic nature of manufacturing workflows and the perception of the complexity of scheduling are quite low in job shops. The most interesting aspect of production scheduling is that a scheduler can somehow ensure the workflow by making real-time common sense decisions (by pushing each job from one workstation to the next based on the progress of the work) or by using one of the methods described above, while researchers consider scheduling in job shops to be highly complex and try to find good solutions in an acceptable time. We do not know how far each of the approaches described above is from the unknown best. It is the simultaneous production of different jobs (with small quantities) using shared resources that makes job shop scheduling quite complex. With the rapid advances in information technology, FCS may have a brighter future for job shop scheduling if it can also accommodate system changes. We are finding more and more FCS applications in job shops where orders are tracked on the shop floor for better production control. Lean methodology should not just mean whatever TPS practises. FCS can be a perfect complement to the Lean toolkit for job shops. It can provide comprehensive production control of complex job shops that avoids the wild generalisations of Kanban control.

In this chapter, we review job shop scheduling problems, discuss various approaches, and give some examples of recent developments in the scheduling literature. We can conclude that, in particular, studies on metaheuristic algorithms are 
rapidly increasing. Our future research will involve case study applications with real data and analysis of the results.

\section{References}

Blazewicz, J.; Dror, M. \& Weglarz, J. (1991). Mathematical-programming formulations for machine scheduling - a survey. European Journal of Operational Research, Vol. 51, No. 3, pp. 283-300, ISSN 0377-2217

Blum, C. \& Roli, A. (2003). Metaheuristics in combinatorial optimization: overview and conceptual comparison. ACM Computing Surveys, Vol. 35, No. 3, pp. 268-308, ISSN 0360-0300

Buchmeister, B.; Palcic, I. \& Ojstersek, R. (2019). Manufacturing scheduling performance - a case study, Proceedings of the 30th International DAAAM Symposium, Katalinic, B. (Ed.), pp. 20-29, ISBN 978-3-902734-22-8, Zadar, Croatia, October 2019, DAAAM International, Vienna

Callefi, J. S. \& Crubellate, J. M. (2020). Toyota Production System and communicative institutionalism and organizational culture. Revista Gestao \& Tecnologia - Journal of Management and Technology, Vol. 20, No. 1, pp. 258-277, ISSN 1677-9479

Carvalho, A. N.; Scavarda, L. F. \& Lustosa, L. J. (2014). Implementing finite capacity production scheduling: lessons from a practical case. International Journal of Production Research, Vol. 52, No. 4, pp. 1215-1230, ISSN 0020-7543

Chakrabortty, R. K.; Rahman, H. F.; Haque, K. M. A.; Paul, S. K. \& Ryan, M. J. (2021). An event-based reactive scheduling approach for the Resource Constrained Project Scheduling Problem with unreliable resources. Computers \& Industrial Engineering, Vol. 151, Paper ID 106981, pp. 1-16, ISSN 0360-8352

Chen, S.; Fang, S. \& Tang, R. (2020). An ANN-based approach for real-time scheduling in cloud manufacturing. Applied Sciences, Vol. 10, No. 7, Paper ID 2491, pp. 1-22, eISSN 2076-3417

Cheung, J. Y. (1994). Scheduling, In: Dagli, C. H. (Ed.), Artificial Neural Networks for Intelligent Manufacturing, Chapter 8, pp. 159-193, Springer Science+Business Media, ISBN 978-0-412-48050-8, Dordrecht

Dave, M. \& Choudhary, K. (2016). Job shop scheduling algorithms - a shift from traditional techniques to non-traditional techniques, 3rd International Conference on Computing for Sustainable Global Development (INDIACom), Hoda, M. N. (Ed.), pp. 169-173, ISBN 978-9-3805-4419-9, New Delhi, India, March 2016, IEEE, New York De Castro, L. N. (2002). Immune, swarm and evolutionary algorithms Part I: basic models, Proceedings of the 9th International Conference on Neural Information Processing, Singapore, Wang, L.; Rajapakse, J. C.; Fukushima, K.; Lee, S.-Y. \& Yao, X. (Eds.), pp. 1464-1468, ISBN 981-04-7524-1, November 2002, IEEE, Piscataway Denkena, B.; Dittrich, M. A.; Stamm, S. C. \& Prasanthan, V. (2019). Knowledge-based process planning for economical re-scheduling in production control, 52nd CIRP Conference on Manufacturing Systems, Butala, P.; Govekar, E. \&Vrabic, R. (Eds.), pp. 980-985, ISBN 978-1713800958, Ljubljana, Slovenia, June 2019, Elsevier, Amsterdam 
Dorigo, M.; Maniezzo, V. \& Colorni, A. (1991). Positive Feedback as a Search Strategy, Technical Report, No. 91-016, pp. 1-20, Politecnico di Milano, Milano Gavriluta, A. (2019). Study on improvement of a manufacturing system using Lean Manufacturing. Quality - Access to Success, Vol. 20, Suppl. 1, pp. 365-370, ISSN 1582-2559

Gen, M. \& Cheng, R. (2000). Genetic Algorithms and Engineering Optimization, John Wiley \& Sons, ISBN 978-0471315315, New York

Glover, F. (1989). Tabu search: part I. ORSA Journal on Computing, Vol. 1, No. 3, pp. 190-206, ISSN 1091-9856

Goldratt, E. M. \& Cox, J. (1986). The Goal: A Process of Ongoing Improvements, North River Press, ISBN 978-0-88427-061-0, Great Barrington, MA, USA

Gupta, J. N. D.; Palanimuthy, N. \& Chen, C. L. (1999). Designing a tabu search algorithm for the twostage flowshop problem with secondary criterion. Production Planning \& Control, Vol. 10, No. 3, pp. 251-265, ISSN 0953-7287

Jarboui, B.; Ibrahim, S.; Siarry, P. \& Rebai, A. (2008). A combinatorial particle swarm optimization for solving permutation flowshop problems. Computers \& Industrial Engineering, Vol. 54, No. 3, pp. 526-538, ISSN 0360-8352

Kong. F. \& Dou, D. (2021). Resource-Constrained Project Scheduling Problem under multiple time constraints. Journal of Construction Engineering and Management, Vol. 147, No. 2, Paper ID 04020170, ISSN 0733-9364

Lendinez, L. C. (2019). Kanban. Methodology to increase process efficiency. 3C Tecnologia, Vol. 8, No. 1, pp. 30-40, ISSN 2254-4143

Lopez-Santana, E. R. \& Mendez-Giraldo, G. A. (2016). A knowledge-based expert system for scheduling in services systems. Applied Computer Sciences in Engineering, Vol. 657, pp. 212-224, ISSN 1865-0929

Low, C.; Yeh, J. Y. \& Huang, K. I. (2004). A robust simulated annealing heuristic for flowshop scheduling problems. International Journal of Advanced Manufacturing Technology, Vol. 23, No. 9-10, pp. 762-767, ISSN 0268-3768

Nagano, M. S.; Ruiz, R. \& Lorena, L. A. N. (2008). A constructive genetic algorithm for permutation flowshop scheduling. Computers \& Industrial Engineering, Vol. 55, No. 1, pp. 195-207, ISSN 0360-8352

Ogbu, F. A. \& Smith, D. K. (1991). Simulated annealing for the permutation flowshop problem. Omega, Vol. 19, pp. 64-67, ISSN 0305-0483

Oh, H. J. A.; Muriel, A. \& Balasubramanian, H. (2014). A user-friendly Excel simulation for scheduling in primary care practices, Proceedings of the 2014 Winter Simulation Conference, Tolk, A.; Yilmaz, L.; Diallo, S. Y. \& Ryzhov, I. O. (Eds.), pp. 1177-1185, ISBN 978-1-4799-7486-3, Savannah, GA, USA, December 2014, IEEE, New York

Ojstersek, R.; Tang, M. \& Buchmeister, B. (2020). Due date optimization in multiobjective scheduling of flexible job shop production. Advances in Production Engineering \& Management, Vol. 15, No. 4, pp. 481-492, ISSN 1854-6250

Onwubolu, G. C. (2002). Emerging Optimization Techniques in Production Planning and Control, Imperial College Press, ISBN 978-1860942662, London 
Parveen, S. \& Ullah, H. (2010). Review on job-shop and flow-shop scheduling using multi criteria decision making. Journal of Mechanical Engineering, Vol. ME 41, No. 2, pp. 130-146, ISSN 0379-4318

Qian, B.; Wang, L.; Huang, D. X.; Wang, W. L. \& Wang, X. (2009). An effective hybrid DE-based algorithm for multi-objective flowshop scheduling with limited buffers. Computers and Operations Research, Vol. 36, No. 1, pp. 209-233, ISSN 03050548

Rabelo, L. C. (1990). A Hybrid Artificial Neural Networks and Knowledge-Based Expert Systems Approach to Flexible Manufacturing System Scheduling, PhD. Dissertation, University of Missouri - Rolla, Rolla

Rajendran, C. \& Ziegler, H. (2004). Ant-colony algorithms for flowshop scheduling to minimize makespan/total flowtime of jobs. European Journal of Operational Research, Vol. 155, No. 2, pp. 426-438, ISSN 0377-2217

Saravanan, M.; Haq, A. N.; Vivekraj, A. R. \& Prasad, T. (2008). Performance evaluation of the scatter search method for permutation flowshop sequencing problems. International Journal of Advanced Manufacturing Technology, Vol. 37, No. 11-12, pp. 1200-1208, ISSN 0268-3768

Tasgetiren, M. F.; Liang, Y. C.; Sevkli, M. \& Gencyilmaz, G. (2007). A particle swarm optimization algorithm for makespan and total flowtime minimization in the permutation flowshop sequencing problem. European Journal of Operational Research, Vol. 177, No. 3, pp. 1930-1947, ISSN 0377-2217

T'kindt, V. \& Billaut, J.-C. (2006). Multicriteria Scheduling, 2nd edition, Springer, ISBN 978-3-540-28230-3, Berlin

Velaga, P. (Optisol.biz) (2018). Various Approaches to Production Scheduling in Job Shops, Available from: https://optisol.biz/job_shop_scheduling.html, Accessed: 201808-25

Wang, L. \& Zhang, L. (2006). Determining optimal combination of genetic operators for flow shop scheduling. International Journal of Advanced Manufacturing Technology, Vol. 30, No. 3-4, pp. 302-308, ISSN 0268-3768

Yagmahan, B. \& Yenisey, M. M. (2009). Scheduling practice and recent developments in flow shop and job shop scheduling, In: Chakraborty, U. K. (Ed.), Computational Intelligence in Flow Shop and Job Shop Scheduling, pp. 261-300, Springer-Verlag, ISBN 978-3-642-02835-9, Berlin

Ying, K. C. \& Liao, C. J. (2004). An ant colony system for permutation flowshop sequencing. Computers and Operations Research, Vol. 31, No. 5, pp. 791-801, ISSN 0305-0548

Yu, J.-M. \& Lee, D.-H. (2018). Scheduling algorithms for job-shop-type remanufacturing systems with component matching requirement, Computers \& Industrial Engineering, Vol. 120, pp. 266-278, ISSN 0360-8352

Zhang, H. \& Zhang, Y. Q. (2020). A discrete job-shop scheduling algorithm based on improved genetic algorithm. International Journal of Simulation Modelling, Vol. 19, No. 3, pp. 517-528, ISSN 1726-4529

Zhang, R. \& Wu, C. (2010). A hybrid immune simulated annealing algorithm for the job shop scheduling problem. Applied Soft Computing, Vol. 10, No. 1, pp. 79-89, ISSN $1568-4946$ 